\title{
Effects of the Polo-like-kinase-1-inhibitor BI2536 in squamous cell carcinoma cell lines of the head and neck
}

\author{
JENS WAGENBLAST ${ }^{1}$, DANIEL HIRTH $^{1}$, LAURA THRON $^{1}$, CHRISTOPH ARNOLDNER $^{2}$, \\ MARC DIENSTHUBER $^{1}$, TIMO STÖVER ${ }^{1}$ and MARKUS HAMBEK ${ }^{1}$ \\ ${ }^{1}$ ENT Department, Medical School, Goethe University, D-60590 Frankfurt am Main, Germany; \\ ${ }^{2}$ ENT Department, Medical University of Vienna, A-1090 Vienna, Austria
}

Received February 10, 2012; Accepted April 19, 2012

DOI: $10.3892 / \mathrm{ol} .2012 .700$

\begin{abstract}
Inhibition of the Polo-like-kinase-1 (PLK1) has been shown to be effective in a number of solid tumor models. In this in vitro study, we examined the antitumor effect of BI2536, a small molecule inhibitor of PLK1, in squamous cell carcinoma of the head and neck (SCCHN) cell lines. Dose escalation studies were performed with nine SCCHN cell lines using BI2536. Growth inhibitory and proapoptotic effects were measured quantitatively using cytohistology and a Human Apoptosis Array Kit. BI2536 demonstrated a significant antiproliferative and apoptotic activity in all nine SCCHN cell lines investigated $(\mathrm{p}<0.009)$. Our results indicate that inhibition of PLK1 by BI2536 leads to an antiproliferative and apoptotic effect in SCCHN cell lines. In vivo and in the clinical setting, the application of BI2536 may support the antitumoral activity of conventional drugs that are in current use and could decrease the systemic toxicity of these drugs.
\end{abstract}

\section{Introduction}

Cancer of the head and neck is one of the most common types of cancer worldwide. Each year more than 600,000 new diagnoses are made worldwide, with the most common sites being the oral cavity, the larynx and the pharynx (1). Although outcomes can differ between tumors of the same stage, the prognosis of patients suffering from head and neck cancer is mainly dependent on the tumor stage, particularly the lymph node status. Besides tobacco and alcohol abuse, an oropharyngeal infection with high-risk human papillomavirus, such as HPV 16 and HPV 18, may lead to the development of a squamous cell carcinoma, particularly of organs of the oral cavity such as the tonsils, tongue and palate (2). Based on the fact that

Correspondence to: Dr Jens Wagenblast, ENT Department, University Hospital, Theodor-Stern-Kai 7, D-60590 Frankfurt/Main, Germany

E-mail: jens.wagenblast@kgu.de

Key words: BI2536, squamous cell head and neck carcinoma cell lines, growth inhibition, apoptosis primarily younger patients suffer from HPV-induced cancer of the head and neck, it seems as if high-risk HPV presents an additional risk factor.

Although surgical techniques and conservative drug therapy strategies have become more efficient in the last decades, prognosis of this type of cancer remains poor. As such, finding new efficient treatment strategies with a long-term beneficial effect is a great challenge. Thus, new antineoplastic drugs must be found and evaluated in vitro and in vivo (3). Fine-mapping of the cell-division cycle, particularly the identification of mitotic kinase signaling pathways, offers new opportunities for cancerdrug development. Polo-like kinase 1 (PLK1) is a major mitotic regulator overexpressed in numerous solid and hematopoietic tumors (4). Moreover, PLK1 plays a key role in the cell cycle at the $\mathrm{G} 2 / \mathrm{M}$ transition and in mitosis (5) and is a negative prognostic marker in specific human cancer types (6-8). In this study, we show the first results obtained with BI2536, a potent small-molecule inhibitor of mammalian PLK1, that inhibits PLK1 activity at low nanomolar concentrations. The aim of the present study was to systematically investigate the growth inhibitory and apoptotic activity of BI2536 as a single agent in nine squamous carcinoma cell lines.

\section{Materials and methods}

Materials. Nine different squamous carcinoma cell lines, mainly of head and neck origin, were tested in this study. A-431 cells were obtained from American Type Culture Collection (ATCC; Manassas, VA, USA). PE/CA-PJ 15, PE/ CA-PJ 41, PE/CA-PJ 49 and PE/CA-PJ 34 cells were obtained from ECACC (European Collection of Cell Cultures), and Cal-27 and Kyse-140 cells from DSMZ GmbH, Braunschweig, Germany. CLS-354 and UM-SCC-14C cells were obtained from Cell Lines Service (CLS, Eppelheim, Germany). The fibroblast cell line was a gift from the Department of Dermatology, University Hospital, Frankfurt/Main, Germany.

Methods. BI2536 was provided by Boehringer Ingelheim $\mathrm{GmbH}$, Austria. Squamous carcinoma cell lines were cultivated according to the instructions of the suppliers with antibiotics at $37^{\circ} \mathrm{C}$ in the cell type-specific medium Quantum 263 with L-glutamine (PAA Laboratories GmbH, Pasching, Austria). Cells were seeded in 96-multiwell plates 


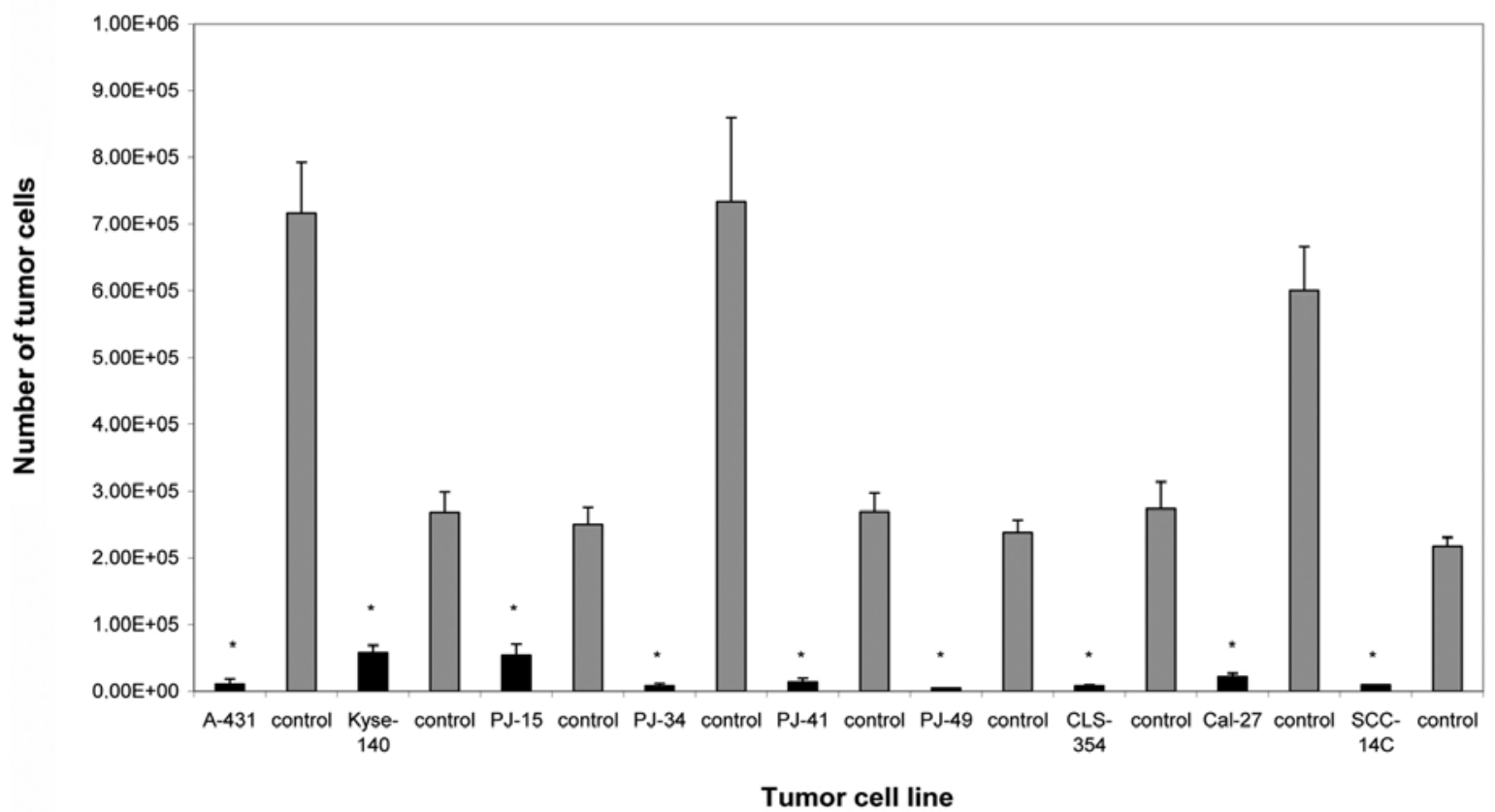

Figure 1. Effect of PLK1 inhibitor BI2536 in nine different squamous cell carcinoma cell lines versus untreated control. Growth inhibitory effect of the single agent BI2536 at a fixed cell line-specific concentration of $2.5 \mathrm{nmol} / \mathrm{l}$ after $72 \mathrm{~h}$ in nine different squamous carcinoma cell lines (black columns) is shown. The corresponding untreated tumor cell lines (grey columns) served as controls and were incubated according to the instructions of the suppliers with antibiotics at $37^{\circ} \mathrm{C}$ in the cell type-specific medium Quantum 263 with L-glutamine. The number of absolute tumor cells in the treated and control cell lines was determined in a Rosenthal chamber at $72 \mathrm{~h}$ after treatment or incubation with Quantum 263 (controls), respectively. The mean values of three independent experiments with standard deviation are shown. Single agent differences versus untreated controls are indicated by asterisks. BI2536 inhibited cell proliferation significantly in all nine cell lines treated $(\mathrm{p}<0.009$ versus controls for all nine cell lines).

(1x10 $0^{5}$ cells/well). Following incubation for $24 \mathrm{~h}$, the cells were treated with BI2536 for 24, 48 and $76 \mathrm{~h}$, respectively. In the experiments described in this study, BI2536 was used in each cell line at a fixed, cell line-specific concentration that had produced maximum growth inhibition in previous systematic investigations in our laboratory. The concentration for the cell lines investigated was $2.5 \mathrm{nmol} / \mathrm{l}$ for BI2536, which demonstrated maximal growth inhibition in our previous dose escalation studies (unpublished data). The number of cells was determined by counting cells in a Rosenthal chamber at 24, 48 and $72 \mathrm{~h}$ subsequent to treatment. Apoptosis was detected by microscopic cytohistology as well as by a Human Apoptosis Array Kit (R\&D Systems, Abingdon, UK) as previously described by other groups $(9,10)$ (Fig. 2).

Statistical analysis. Each experiment was carried out in triplicate. For statistical analysis, a Wilcoxon test for matched pairs (dependent samples) was performed using SPSS 19.0 software for Windows. Differences of $\mathrm{p}<0.05$ were considered statistically significant.

\section{Results}

Nine different squamous carcinoma cell lines, mainly of head and neck origin, were tested in this study. Following $24 \mathrm{~h}$ incubation, the cells were treated with BI2536 for 24, 48 and $76 \mathrm{~h}$, respectively. Compared with the untreated control groups, the PLK1 inhibitor BI2536 had a highly significant antiproliferative and apoptotic effect in all nine tumor cell lines $(\mathrm{p}<0.009)$ (Fig. 1). Apoptosis was detected by microscopic cytohistology

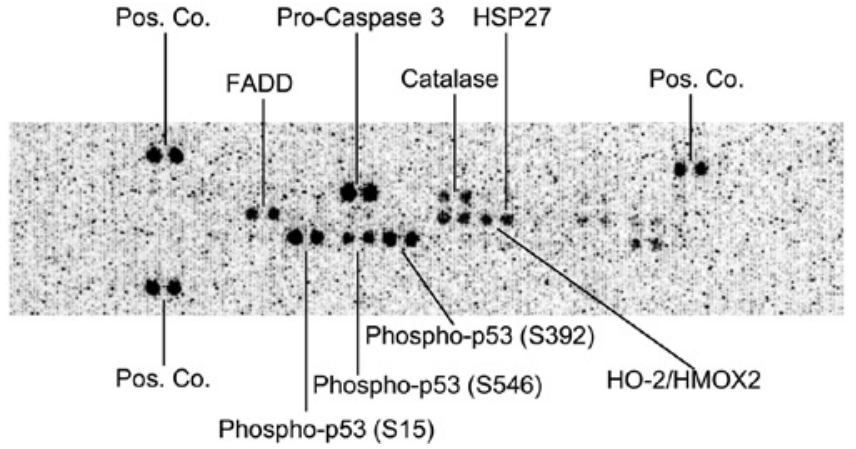

Figure 2. Detection of pro-caspase 3 as a typical apoptosis marker by a Human Apoptosis Array Kit. Besides other cell markers, the typical apoptotic marker pro-caspase 3 was detected in nine squamous carcinoma cell lines treated with BI2536. The results obtained when Kyse-140 cells were treated with BI2536 are shown. Pos.co, positive control.

as well as by a Human Apoptosis Array Kit (R\&D Systems). Additionally, pro-caspase 3 was detected as a typical apoptotic marker (Fig. 2). BI2536 is therefore a potent small-molecule inhibitor of mammalian PLK1 that inhibits PLK1 activity at low nanomolar concentrations leading to an apoptotic effect in nine squamous carcinoma cell lines.

\section{Discussion}

Previously, a growing body of biochemical investigations contributed to the improved characterization of biological 
tumor behaviour, thereby improving treatment strategies. PLK1 is a marker of cell proliferation that shows an elevated expression in a number of solid tumors $(11,12)$. Previous studies have been able to demonstrate that the level of PLK1 correlates with metastasis in cervical lymph nodes (13). Moreover, there was a significantly higher occurrence of PLK expression in metastatic HNSCC than in non-metastatic tumors. This observation suggests that the determination of PLK expression in primary tumors of the head and neck indicates the metastasizing potential of a tumor (13). Patients could be subdivided by PLK expression into those with better or worse prognosis, suggesting PLK levels as a discriminatory marker within conventional tumor stages (13). Despite the development of multimodal treatment strategies in head and neck cancer disease, the overall 5-year survival rate in stages UICC III and IV remains poor (14). As such, finding new efficient treatment strategies with long-term beneficial effects is necessary. Thus, new antineoplastic drugs that show less toxicity but a high antineoplastic potency should be evaluated in vitro and in vivo $(3,15)$.

The dihydropteridinone derivate BI2536 is a potent small-molecule inhibitor of mammalian PLK1 that inhibits PLK1 activity at low nanomolar concentrations. BI2536 induces $\mathrm{G} 2 / \mathrm{M}$ arrest and the formation of abnormal mitotic figures, such as monopolar spindles. Although there are already clinical phase I and II trials conducted in which BI2536 is administered in different tumor entities there remains a need for further in vitro studies focusing on the pharmacological profile of BI2536 (16). In the present study, we systematically evaluated the effect of BI2536 in nine different squamous cell carcinoma cell lines for the first time. Compared with the untreated control groups, BI2536 had a highly significant antiproliferative and apoptotic effect in all nine tumor cell lines. Taken together, based on our promising results, BI2536 may be of great interest in future treatment regimes used for head and neck cancer.

In conclusion, PLK1 is a major mitotic regulator overexpressed in various solid and hematopoietic tumors. In the present study, BI2536, a potent small-molecule inhibitor of mammalian PLK1 demonstrated, when applied as a single agent, a strong inhibition of cell proliferation accompanied by enhanced apoptosis in nine different squamous carcinoma cell lines. Introducing drugs that block PLK1 to head and neck cancer therapy may improve adjuvant or non-surgical conservative treatment of this tumor identity. More studies are required to prove whether there is an enhancing antitumoral effect of BI2536 in combination with docetaxel or cisplatin that are, among other drugs such as paclitaxel and 5-fluorouracil, commonly used in the modern treatment of head and neck cancer. Our results emphasize that targeting the PLK signaling pathway is a potentially noteworthy therapeutic target for the modern treatment of head and neck cancer disease.

\section{Acknowledgements}

We thank Erika Weith for her excellent technical support. We also thank Boehringer Ingelheim for their donation of BI2536.

\section{References}

1. Mehanna H, Paleri V, West CM and Nutting C: Head and neck cancer - Part 1: epidemiology, presentation, and prevention. BMJ 341: c4684, 2010.

2. Maden C, Beckmann AM, Thomas DB, McKnight B, Sherman KJ, Ashley RL, et al: Human papillomaviruses, herpes simplex viruses, and the risk of oral cancer in men. Am J Epidemiol 135: 1093-1102, 1992.

3. Wagenblast J, Hambek M, Baghi M, Gstottner W, Strebhardt K, Ackermann $\mathrm{H}$, et al: Antiproliferative activity of bortezomib alone and in combination with cisplatin or docetaxel in head and neck squamous cell carcinoma cell lines. J Cancer Res Clin Oncol 134: 323-330, 2008.

4. Renner AG, Dos Santos C, Recher C, Bailly C, Creancier L, Kruczynski A, et al: Polo-like kinase 1 is overexpressed in acute myeloid leukemia and its inhibition preferentially targets the proliferation of leukemic cells. Blood 114: 659-662, 2009.

5. Van de Weerdt BC and Medema RH: Polo-like kinases: a team in control of the division. Cell Cycle 5: 853-864, 2006.

6. Steegmaier M, Hoffmann M, Baum A, Lenart P, Petronczki M, Krssak M, et al: BI 2536, a potent and selective inhibitor of polo-like kinase 1, inhibits tumor growth in vivo. Curr Biol 17: 316-322, 2007.

7. Takai N, Hamanaka R, Yoshimatsu J and Miyakawa I: Polo-like kinases (Plks) and cancer. Oncogene 24: 287-291, 2005.

8. Strebhardt K and Ullrich A: Targeting polo-like kinase 1 for cancer therapy. Nat Rev Cancer 6: 321-330, 2006.

9. Datta D, Banerjee P, Gasser M, Waaga-Gasser AM and Pal S: CXCR3-B can mediate growth-inhibitory signals in human renal cancer cells by down-regulating the expression of heme oxygenase-1. J Biol Chem 285: 36842-36848, 2010.

10. Ray RB, Raychoudhuri A, Steele R and Nerurkar P: Bitter melon (Momordica charantia) extract inhibits breast cancer cell proliferation by modulating cell cycle regulatory genes and promotes apoptosis. Cancer Res 70: 1925-1931, 2010.

11. Holtrich U, Wolf G, Brauninger A, Karn T, Bohme B, Rubsamen-Waigmann $\mathrm{H}$, et al: Induction and down-regulation of PLK, a human serine/threonine kinase expressed in proliferating cells and tumors. Proc Natl Acad Sci USA 91: 1736-1740, 1994

12. Wolf G, Elez R, Doermer A, Holtrich U, Ackermann H, Stutte HJ, et al: Prognostic significance of polo-like kinase (PLK) expression in non-small cell lung cancer. Oncogene 14: 543-549, 1997.

13. Knecht R, Elez R, Oechler M, Solbach C, von Ilberg C and Strebhardt K: Prognostic significance of polo-like kinase (PLK) expression in squamous cell carcinomas of the head and neck. Cancer Res 59: 2794-2797, 1999.

14. Jemal A, Murray T, Ward E, Samuels A, Tiwari RC, Ghafoor A, et al: Cancer statistics. CA Cancer J Clin 55: 10-30, 2005.

15. Wagenblast J, Baghi M, Arnoldner C, Bisdas S, Gstottner W, Ackermann $\mathrm{H}$, et al: Cetuximab enhances the efficacy of bortezomib in squamous cell carcinoma cell lines. J Cancer Res Clin Oncol 135: 387-393, 2009.

16. Schoffski P, Blay JY, De Greve J, Brain E, Machiels JP, Soria JC, et al: Multicentric parallel phase II trial of the polo-like kinase 1 inhibitor BI 2536 in patients with advanced head and neck cancer, breast cancer, ovarian cancer, soft tissue sarcoma and melanoma. The first protocol of the European Organization for Research and Treatment of Cancer (EORTC) Network Of Core Institutes (NOCI). Eur J Cancer 46: 2206-2215, 2010. 